\title{
Macrolides: A Canadian Infectious Disease Society position paper
}

\author{
S McKenna BScPhm ${ }^{1}$, GA Evans $\mathrm{MD}^{2}$ and the \\ Canadian Infectious Disease Society Antimicrobial Agents Committee*
}

\begin{abstract}
S McKenna, GA Evans and the Canadian Infectious Disease Society Antimicrobial Agents Committee. Macrolides: A Canadian Infectious Disease Society position paper. Can J Infect Dis 2001;12(4):218-231.

Since the introduction of erythromycin in 1965, no new compounds from the macrolide antimicrobial class were licensed in Canada until the 1990s. Clarithromycin and azithromycin, since their introduction, have become important agents for treating a number of common and uncommon infectious diseases. They have become prime agents in the treatment of respiratory tract infections, and have revolutionized the management of both genital chlamydial infections, by the use of single-dose therapy with azithromycin, and nontuberculous mycobacterial infections, by the use of clarithromycin. The improvement of clarithromycin and azithromycin over the gastrointestinal intolerability of erythromycin has led to supplanting the use of the latter for many primary care physicians. Unfortunately, the use of these agents has also increased the likelihood for misuse and has raised concerns about a resultant increase in the rates of macrolide resistance in many important pathogens, such as Streptococcus pneumoniae. This paper reviews the pharmacology and evidence for the current indications for use of these newer agents, and provides recommendations for appropriate use.
\end{abstract}

Key Words: Azithromycin; Clarithromycin; Erythromycin; Macrolides; Review; Therapeutic use

\section{Macrolides : Énoncé de position de la Société canadienne des maladies infectieuses}

RÉSUMÉ : Depuis l'introduction de l'érythromycine en 1965, aucune nouvelle molécule de la classe des macrolides n'a été brevetée, soit depuis les années 1990. Depuis leur lancement, la clarithromycine et l'azithromycine sont devenues d'importants agents thérapeutiques pour de nombreuses maladies infectieuses courantes ou rares. On les utilise en effet comme agents de premier recours pour le traitement des infections respiratoires et elles ont révolutionné le traitement des infections génitales à Chlamydia par le recours à des posologies monodoses avec azythromycine, et les infections mycobactériennes non tuberculeuses par le recours à la clarithromycine. L'amélioration que représentent la clarithromycine et l'azythromycine par rapport à l'érythromycine, dont les effets secondaires gastro-intestinaux sont importants, a relégué cette dernière au second rang pour de nombreux omnipraticiens. Malheureusement, l'emploi de ces agents a également fait augmenter le risque de leur utilisation à mauvais escient et soulève des problèmes d'augmentation des taux de résistance aux macrolides que manifestent de nombreux agents pathogènes importants, comme Streptococcus pneumoniae. Cet article passe en revue les aspects pharmacologiques et les preuves sur lesquelles s'appuient les indications actuelles des agents plus récents et formule des recommandations pour leur utilisation appropriée.

${ }^{1}$ Department of Pharmacy Services, Kingston General Hospital, Kingston, Ontario; ${ }^{2}$ Division of Infectious Diseases, Department of Medicine, Queen's University, Kingston, Ontario

*Members: Drs Susan King, Toronto, Ontario; Coleman Rotstein, Hamilton, Ontario; Michel Laverdiere, Montreal, Quebec; Peter Phillips, Vancouver, British Columbia

Declared conflicts: Dr Evans has received research support and speaking honoraria from Abbott Laboratories Ltd and Pfizer Canada Inc Correspondence: GA Evans, Division of Infectious Diseases, Room 3013, Etherington Hall, Queen's University, 94 Stuart Street, Kingston,

Ontario K7L 3N6. Telephone 613-533-6619, fax 613-533-6695, e-mail gae@post.queensu.ca

Received for publication April 23, 2001. Accepted April 23, 2001 
$\mathrm{E}$ rythromycin A is a naturally occurring, microbiologically active compound of the macrolide class of antibiotics. Chemical modification of erythromycin A's 14-membered lactone ring has led to the formation of semisynthetic derivatives with not only improved bioavailability and tolerability, but also expanded spectrums of microbiological activity and improved pharmacokinetic profiles. Such modifications produced clarithromycin, classified as a macrolide because it retains the central 14-membered lactone ring $(1,2)$, and azithromycin, classified as an azalide due to its 15-membered aglycone ring (1). The latter two compounds are the newest agents in the macrolide class licensed for use in Canada. Roxithromycin and dirithromycin are available in other countries.

These compounds are clinically active against Gram-positive and Gram-negative cocci, and Gram-negative bacilli (primarily Haemophilus influenzae, Legionella species, Moraxella catarrhalis, Campylobacter jejuni, Bordatella pertussis and Helicobacter pylori). Azalides such as azithromycin have exhibited superior activity against Gramnegative pathogens and are generally less active against Gram-positive pathogens. Intracellular pathogens such as Chlamydia species, Mycoplasma species, Ureaplasma species, Borrelia species and nontuberculous mycobacteria species show varying susceptibilities. On the basis of their microbial activity, both the macrolides and azalides have been shown to be clinically useful in the treatment of uncomplicated skin and soft tissue infections, upper and lower respiratory tract infections, sexually transmitted Chlamydia trachomatis infection and peptic ulcer disease. Additionally, the improved pharmacokinetic profiles and acid stability exhibited by the newer agents may lead to enhanced patient adherence through less frequent dosing and improved bioavailability in the presence of food.

\section{MECHANISMS OF ACTION AND RESISTANCE}

Macrolides and azalides exert their antimicrobial activity by inhibiting translocation of aminoacyl transfer RNA through reversible binding to the $50 \mathrm{~S}$ ribosomal subunit. In this manner, bacterial protein synthesis is inhibited (2). Generally, these agents are considered to be bacteriostatic. However, bactericidal activity has been demonstrated in vitro against certain strains of Streptococcus pyogenes and Streptococcus pneumoniae. Additionally, clarithromycin (and its active 14-hydroxy metabolite) and azithromycin have exhibited bactericidal activity against $H$ influenzae (2). The antibacterial activity of clarithromycin and azithromycin is most likely enhanced by unique tissue distribution and elimination profiles, which result in high and sustained tissue levels relative to those produced with the use of erythromycin (3). These pharmacokinetics provide a probable explanation for the enhanced clinical activity of the newer agents against intracellular pathogens such as Chlamydia species $(2,3)$.

Microbial resistance to macrolides and azalides may be either intrinsic or acquired in nature (4). At physiological pH, macrolides exhibit a low degree of ionization. The intrinsic resistance of the vast majority of Gram-negative bacilli and enterococci to these hydrophobic drugs is believed to be due to outer membrane impermeability (2). Acquired resistance occurs most frequently via either the induction of enzymes causing ribosomal methylation, a target site modification or active efflux. Ribosomal target site modification is mediated by one of a number of erm genes (erm $\mathrm{A}$, erm $\mathrm{B}$, erm $\mathrm{C}, \mathrm{erm} \mathrm{TR}$ ). These genes modify the ribosome through methylation of a specific adenine residue in a conserved region of the $23 \mathrm{~S}$ ribosome that interacts with macrolide, lincosamide and streptogramin type $\mathrm{B}$ antibiotics, conferring the so-called ' $\mathrm{LLS}_{\mathrm{B}}$ ' phenotype (5). Macrolide, lincosamide and streptogramin (MLS) resistance typically results in high levels of resistance to all macrolides leading to erythromycin minimum inhibitory concentrations (MICs) of $64 \mathrm{mg} / \mathrm{L}$ or greater. Active efflux of macrolides and azalides has been demonstrated in both staphylococci and streptococci, and generally confers low level resistance to these drugs $(4,6)$. In Staphylococcus aureus, efflux is mediated through $m s r A$ and $m s r B$ genes encoding an adenosine triphosphate-dependent efflux pump, and results in both macrolide and streptogramin resistance or the MS phenotype. In $S$ pneumoniae and $S$ pyogenes, the genes mefE and $m e f A$, respectively, encode for two transporter efflux pumps (MefE and MefA) that confer resistance only to macrolides and azalides (6). The latter is referred to as the M phenotype. Macrolide modification or inactivation (eg, Escherichia coli production of erythromycin esterase) does occur but is rare in $S$ aureus, and has not been described in $S$ pneumoniae or $S$ pyogenes. Macrolide resistance rates in Canada remain low and have not exceeded $10 \%$ among isolates of $S$ pneumoniae (6). In a 1999 Canadian study of 121 macrolide-resistant $S$ pneumoniae isolates, $50.4 \%$ possessed the M phenotype (mefA), while $45.5 \%$ demonstrated MLS resistance (erm B). Both resistance genes were found in $3.3 \%$ of isolates, while neither genetic determinant was found in $0.8 \%(7)$. In North America, macrolide resistance in $S$ pyogenes remains at less than $3 \%(6)$.

\section{IN VITRO ACTIVITY}

Table 1 lists published MICs of erythromycin, clarithromycin and its active metabolite, where relevant, and azithromycin, for pathogens of clinical interest. Streptococci are usually susceptible to the macrolides and azalides; however, erythromycin and clarithromycin are generally more active than azithromycin. As noted previously, susceptibility of staphylococcal strains is extremely variable due to several possible mechanisms of resistance.

In certain cases, the in vivo activity of clarithromycin and azithromycin against Gram-negative and intracellular pathogens has been shown to be better than anticipated on the basis of in vitro data. For example, the activity of clarithromycin against $H$ influenzae is enhanced in vivo by the microbiological activity of its metabolite, 14-hydroxyclarithromycin. Other factors that may confound the interpretation of in vitro MIC data include the postulated postantibiotic effect, the effect of serum and the variability of activity according to the $\mathrm{pH}$ at the site of action (2). 
TABLE 1

Published minimum inhibitory concentrations (mg/L) of erythromycin, clarithromycin (and 14-hydroxyclarithromycin [14-OH]) and azithromycin for pathogens of clinical interest

\begin{tabular}{|c|c|c|c|}
\hline Pathogen & Erythromycin & Clarithromycin & Azithromycin \\
\hline \multicolumn{4}{|l|}{ Gram-positive cocci } \\
\hline Staphylococcus aureus (susceptible) & 0.06 to 0.25 & $\begin{array}{c}0.03 \text { to } 0.25 \\
\text { (same for } 14-\mathrm{OH} \text { metabolite) }\end{array}$ & 0.06 to 0.5 \\
\hline$S$ aureus (inducible resistance) & 4 to $>128$ & $\begin{array}{c}4 \text { to }>128 \\
\text { (same for } 14-\mathrm{OH} \text { metabolite) }\end{array}$ & 4 to $>128$ \\
\hline$S$ aureus (constitutive resistance) & $>128$ & $\begin{array}{c}>128 \\
\text { (same for } 14-\mathrm{OH} \text { metabolite) }\end{array}$ & $>128$ \\
\hline Streptococcus pneumoniae & 0.01 to 0.06 & $<0.01$ to 0.03 & 0.03 to 0.12 \\
\hline Streptococcus agalactiae & 0.01 to 0.06 & 0.01 to 0.03 & 0.06 to 0.12 \\
\hline Streptococcus pyogenes & 0.01 to 0.03 & $<0.01$ to 0.03 & 0.03 to 0.12 \\
\hline \multicolumn{4}{|l|}{ Gram-negative cocci } \\
\hline Neisseria gonorrhoeae & 0.06 to 1 & 0.06 to 0.5 & 0.03 to 0.06 \\
\hline \multicolumn{4}{|l|}{ Gram-negative bacilli } \\
\hline Haemophilus influenzae & 0.5 to 4 & $\begin{array}{c}1 \text { to } 8 \\
\text { (0.5 to } 4 \text { for } 14-\mathrm{OH} \text { metabolite) }\end{array}$ & 0.25 to 1 \\
\hline Haemophilus ducreyi & 0.001 to 0.01 & No data & 0.001 to 0.004 \\
\hline Moraxella catarrhalis & 0.03 to 0.25 & 0.03 to 0.25 & 0.03 to 0.06 \\
\hline Bordetella pertussis & 0.008 to 0.03 & 0.008 to 0.03 & 0.01 to 0.03 \\
\hline Helicobacter pylori & 0.12 to 0.25 & 0.03 to 0.06 & 0.25 \\
\hline \multicolumn{4}{|l|}{ Intracellular microorganisms } \\
\hline Legionella species & 0.25 to 2 & 0.03 to 0.06 & 0.03 to 0.5 \\
\hline Mycoplasma pneumoniae & 0.008 to 0.03 & 0.002 to 0.008 & $<0.002$ to 0.004 \\
\hline Mycoplasma hominis & $>32$ & 8 to 64 & 2 to 16 \\
\hline Ureaplasma urealyticum & 0.12 to 2 & 0.015 to 0.25 & 0.12 to 1 \\
\hline Chlamydia pneumoniae & 0.06 to 0.125 & $\begin{array}{c}0.015 \text { to } 0.03 \\
(0.015 \text { for } 14-\mathrm{OH} \text { metabolite })\end{array}$ & 0.06 to 1 \\
\hline Chlamydia trachomatis & 0.06 to 1.02 & 0.007 to 0.008 & 0.032 to 1.02 \\
\hline Mycobacterium avium complex* & $>8$ & 0.25 to $>8$ & $>8$ \\
\hline Mycobacterium tuberculosis* & $>8$ & 0.125 to $>8$ & $>8$ \\
\hline Mycobacterium kansasii* & $>8$ & 0.5 to $>8$ & 4 to $>8$ \\
\hline Mycobacterium chelonae* & $>8$ & 0.5 to $>8$ & $>8$ \\
\hline Mycobacterium fortuitum* & 4 to $>8$ & 0.25 to $>8$ & 4 to $>8$ \\
\hline Borrelia burgdorferi & 0.03 to 0.16 & 0.015 to 0.06 & 0.015 to 0.04 \\
\hline
\end{tabular}

Unless otherwise specified, data taken from references 2, 8 and 93. * Data from reference 94

Inherent difficulties in the determination of MICs for intracellular pathogens also emphasize the need for cautious interpretation (8).

Mycoplasma pneumoniae is generally susceptible to the aforementioned macrolides and azithromycin. Both clarithromycin and azithromycin are active against certain pathogens involved in sexually transmitted disease; namely, $C$ trachomatis and Haemophilus ducreyi.

Organisms of the Mycobacterium avium complex (MAC) are susceptible to both clarithromycin and azithromycin. Other nontuberculous mycobacteria, including Mycobacterium kansasii, Mycobacterium simae, Mycobacterium xenopi and Mycobacterium malmoense, have been shown to be susceptible in vitro to both azithromycin and clarithromycin in a beige mouse model of infection (9). Clarithromycin has been shown to possess broad in vitro activity against many nontuberculous mycobacteria, including Mycobacterium scrofulaceum, Mycobacterium szulgai, $M$ xenopi, Mycobacterium fortuitum complex and Mycobacterium marinum (10). Neither new macrolide nor related compounds possess significantly important clinical activity against Mycobacterium tuberculosis.
Clarithromycin exhibits the greatest activity against $H$ pylori, whereas both clarithromycin and azithromycin are active against Borrelia burgdorferi. Clarithromycin and azithromycin are each more active than erythromycin against Legionella pneumophila.

\section{PHARMACOKINETICS}

The oral bioavailability of erythromycin is variable, and depends on the presence of food and formulation administered. Erythromycin degrades rapidly in the presence of acid to form products devoid of antimicrobial activity. Unlike erythromycin, clarithromycin and azithromycin are stable in the presence of acid. Both are readily absorbed after oral administration. Food delays the time to peak serum concentrations of clarithromycin but does not affect the extent of absorption (bioavailability of $55 \%$ ) $(2,11)$. The bioavailability of azithromycin is approximately $37 \%$; however, food can decrease this value by as much as $50 \%$.

Erythromycin attains a peak serum concentration $\left(\mathrm{C}_{\max }\right)$ of $1.8 \mathrm{mg} / \mathrm{L}$ approximately $1.7 \mathrm{~h}$ after oral administration (12). Clarithromycin reaches a $\mathrm{C}_{\max }$ of $2.1 \mathrm{mg} / \mathrm{L}$ after a similar period of time. The peak concentration of azithromycin is 
$0.4 \mathrm{mg} / \mathrm{L}$, achieved approximately $2.5 \mathrm{~h}$ after oral administration. Erythromycin and azithromycin are also available commercially in parenteral formulations. At steady state, the $\mathrm{C}_{\max }$ of parenteral erythromycin, administered in 500 to $1000 \mathrm{mg}$ doses four times daily, is approximately 10 to $17 \mathrm{mg} / \mathrm{L} \mathrm{(13).}$ Azithromycin, administered in $500 \mathrm{mg}$ doses intravenously once daily, reaches a steady state $C_{\max }$ of $3 \mathrm{mg} / \mathrm{L} \mathrm{(14).}$

Clarithromycin and azithromycin are more extensively distributed than erythromycin (volume of distribution of erythromycin $=0.64 \mathrm{~L} / \mathrm{kg}$; volume of distribution of clarithromycin $=$ $3.4 \mathrm{~L} / \mathrm{kg}$; volume of distribution of azithromycin = $23 \mathrm{~L} / \mathrm{kg}$ ) (11). High concomitant serum concentrations are maintained with clarithromycin, while low serum concentrations with concomitant high and persistent tissue concentrations occur with azithromycin. Peak clarithromycin concentrations in the lung have been shown to exceed that in plasma by sixfold. Two- to sixfold tissue to plasma clarithromycin concentrations also occur in the nasal mucosa and tonsils (2). Similarly, azithromycin tissue concentrations are 10- to 100 -fold higher than those in plasma (15). In comparison, erythromycin exhibits a tissue to plasma concentration ratio of only 0.5 - to fivefold (10). Azithromycin becomes highly concentrated in various cells (eg, polymorphonuclear leukocytes, monocytes, alveolar macrophages and fibroblasts) and is subsequently released slowly into the extracellular space. In adults, a single oral $500 \mathrm{mg}$ dose of azithromycin has been shown to produce tissue drug concentrations that are in excess of the minimum inhibitory concentration for many pathogens at sites of infection (bronchial epithelial lining fluid, sputum and bronchial mucosa) for up to $96 \mathrm{~h}$ postdose (12). Tonsillar levels exceed plasma concentrations by over 150-fold up to $84 \mathrm{~h}$ after two oral doses of $250 \mathrm{mg}$ every $12 \mathrm{~h}$ (16).

Clarithromycin and azithromycin exhibit $45 \%$ to $50 \%$ and $7 \%$ to $50 \%$ protein binding, respectively, in human serum compared with $65 \%$ to $90 \%$ for erythromycin base (17). Due to the saturable nature of azithromycin binding, increased free drug concentrations are noted with increased total serum drug concentrations (12).

The elimination half-lives of erythromycin, clarithromycin and azithromycin are $2 \mathrm{~h}, 4 \mathrm{~h}$ and $68 \mathrm{~h}$, respectively, after administration of a single $500 \mathrm{mg}$ oral dose (11). Clarithromycin undergoes extensive hepatic metabolism, and there is a substantial first pass effect. The major metabolic pathway is hydroxylation, which results in the formation of the active 14-hydroxyl metabolite. Both unchanged clarithromycin and its metabolites are eliminated in the feces and the urine. Clarithromycin's pharmacokinetics are nonlinear due to a saturable metabolism; therefore, increased half-life and decreased metabolic clearance occur with increased doses (2). Azithromycin is primarily eliminated unchanged, principally, in the feces and, to a lesser extent, in the urine (12).

\section{CLINICAL TRIALS}

Respiratory tract infections: Several randomized clinical trials have shown clarithromycin and azithromycin to be as effective as erythromycin and other conventional antibiotics in the treatment of upper and lower respiratory tract infections (Table 2).

Upper respiratory tract infections: Clinical response to clarithromycin and azithromycin therapy was found to be similar to the response found with the use of penicillin $\mathrm{V}$ in the treatment of group A beta-hemolytic streptococcal pharyngitis in children (18-20) and adults (21). However, bacteriological cure rates were variable. Compared with penicillin V, eradication rates were higher in clarithromycin-treated groups and lower in azithromycin-treated groups. One study found azithromycin $10 \mathrm{mg} / \mathrm{kg}$ daily for three days to be clinically inferior to penicillin $\mathrm{V} \mathrm{50,000} \mathrm{U} / \mathrm{kg} /$ day administered in two divided doses for 10 days in children with group A betahemolytic streptococcal pharyngitis (22). Clinical response rates comparable with that of penicillin $\mathrm{V}$ have been reported using azithromycin $12 \mathrm{mg} / \mathrm{kg} /$ day daily for five days $(23,24)$.

Acute maxillary sinusitis in adults appears to respond equally well to clarithromycin $500 \mathrm{mg}$ orally twice daily, amoxicillin $500 \mathrm{mg}$ orally three times daily or amoxicillin (combined with clavulanate) $500 \mathrm{mg}$ orally three times daily $(25,26)$. Similarly, an open-label, noncomparative study for this indication demonstrated the effectiveness of azithromycin $500 \mathrm{mg}$ orally on the first day followed by $250 \mathrm{mg}$ orally for four more days (27). Azithromycin $500 \mathrm{mg}$ orally for three days was found to be clinically as effective as amoxicillin/clavulanate $500 \mathrm{mg}$ administered three times daily for 10 days in the treatment of nonsevere, acute maxillary or ethmoidal sinusitis (28).

Clinical response to therapy with azithromycin or amoxicillin/clavulanate was found to be equivalent in the treatment of acute otitis media in paediatric patients (29). However, bacteriological failures with azithromycin recently have been demonstrated. Amoxicillin/clavulanate therapy was associated with higher middle ear fluid bacterial pathogen eradication rates and increased likelihood of clinical improvement in culture-positive patients compared with azithromycin (30). Similarly, bacteriological failure has been demonstrated in a study comparing azithromycin with cefaclor (31). Clinical cure or improvement has been documented in trials comparing clarithromycin and amoxicillin (32,33), amoxicillin/clavulanate $(34,35)$ or cefaclor $(36)$.

Acute exacerbations of chronic bronchitis: Studies of the treatment of mild to moderate acute exacerbations of chronic bronchitis in adults have demonstrated acceptable clinical response rates to clarithromycin administered for five to 10 days or cefaclor for seven days $(37,38)$. Clarithromycin $500 \mathrm{mg}$ has also been compared with oral ciprofloxacin $500 \mathrm{mg}$, each administered twice daily for 14 days in the treatment of acute bacterial exacerbations of bronchitis. Ciprofloxacin therapy was associated with a longer infection-free interval (median 142 days versus 51 days for clarithromycin, $\mathrm{P}=0.15$ ). There were trends toward better clinical response and bacteriological response rates with ciprofloxacin than with clarithromycin (90\% versus $82 \%$ [not significant] and $91 \%$ versus $77 \%[\mathrm{P}=0.01]$, respectively) (39). This finding is in contrast to an earlier trial that demonstrated similar clini- 


\section{TABLE 2}

Comparative clinical trials: Respiratory tract infections

\begin{tabular}{|c|c|c|c|}
\hline Reference & Patient population & Agents compared & Results \\
\hline $\begin{array}{l}\text { Still et al, } \\
1993 \text { (18) }\end{array}$ & $\begin{array}{l}\text { Paediatric } \\
\text { Group A beta-hemolytic } \\
\text { streptococcal pharyngitis } \\
(\mathrm{n}=506)\end{array}$ & $\begin{array}{l}\text { Clarithromycin } 7.5 \mathrm{mg} / \mathrm{kg} \text { by mouth bid for } 10 \text { days } \\
\text { Penicillin VK } 13.3 \mathrm{mg} / \mathrm{kg} \text { by mouth tid for } 10 \text { days }\end{array}$ & $\begin{array}{l}\text { Clinical response } \\
\text { Clarithromycin 96\% } \\
\text { Penicillin VK 94\% (NS) } \\
\text { Bacterial eradication } \\
\text { Clarithromycin 92\% } \\
\text { Penicillin VK } 81 \%(\mathrm{P}=0.004)\end{array}$ \\
\hline $\begin{array}{l}\text { Schaad and Heynen, } \\
1996 \text { (19) }\end{array}$ & $\begin{array}{l}\text { Paediatric } \\
\text { Group A beta-hemolytic } \\
\text { streptococcal pharyngitis } \\
(\mathrm{n}=343)\end{array}$ & $\begin{array}{l}\text { Azithromycin } 10 \mathrm{mg} / \mathrm{kg} \text { by mouth daily for three days } \\
\text { Penicillin } \mathrm{V} 100,000 \mathrm{U} / \mathrm{kg} \text { by mouth tid for } 10 \text { days }\end{array}$ & $\begin{array}{l}\text { Clinical response } \\
\text { Azithromycin 93\% } \\
\text { Penicillin V 90\% (NS) } \\
\text { Bacterial eradication } \\
\text { Azithromycin 55\% } \\
\text { Penicillin V 80\% (P<0.001) }\end{array}$ \\
\hline $\begin{array}{l}\text { McCarty et al, } \\
2000(20)\end{array}$ & $\begin{array}{l}\text { Paediatric } \\
\text { Group A beta-hemolytic } \\
\text { streptococcal pharyngitis } \\
(\mathrm{n}=528)\end{array}$ & $\begin{array}{l}\text { Clarithromycin } 7.5 \mathrm{mg} / \mathrm{kg} \text { by mouth bid for five days } \\
\text { Penicillin VK } 13.3 \mathrm{mg} / \mathrm{kg} \text { by mouth tid for } 10 \text { days }\end{array}$ & $\begin{array}{l}\text { Clinical response } \\
\text { Clarithromycin 97\% } \\
\text { Penicillin VK 94\% (NS) } \\
\text { Bacterial eradication } \\
\text { Clarithromycin 94\% } \\
\text { Penicillin VK 78\% (P<0.001) }\end{array}$ \\
\hline $\begin{array}{l}\text { Bachand, } \\
1991(21)\end{array}$ & $\begin{array}{l}\text { Adult } \\
\text { Group A beta-hemolytic } \\
\text { streptococcal pharyngitis } \\
(n=128)\end{array}$ & $\begin{array}{l}\text { Clarithromycin } 250 \mathrm{mg} \text { by mouth bid } \\
\text { Penicillin VK } 250 \mathrm{mg} \text { by mouth four times daily }\end{array}$ & $\begin{array}{l}\text { Clinical response } \\
\text { Clarithromycin 86\% } \\
\text { Penicillin VK 77\% (NS) } \\
\text { Bacterial eradication } \\
\text { Clarithromycin 88\% } \\
\text { Penicillin VK 91\% (NS) }\end{array}$ \\
\hline $\begin{array}{l}\text { Pacifico et al, } \\
1996(22)\end{array}$ & $\begin{array}{l}\text { Paediatric } \\
\text { Group A beta-hemolytic } \\
\text { streptococcal pharyngitis } \\
(\mathrm{n}=154)\end{array}$ & $\begin{array}{l}\text { Azithromycin } 10 \mathrm{mg} / \mathrm{kg} \text { by mouth daily for three days } \\
\text { Penicillin } \mathrm{V} 50,000 \mathrm{U} / \mathrm{kg} / \text { day bid for } 10 \text { days }\end{array}$ & $\begin{array}{l}\text { Clinical response } \\
\text { Azithromycin } 75 \% \\
\text { Penicillin V 91\% }(\mathrm{P}<0.05) \\
\text { Bacterial eradication } \\
\text { Azithromycin } 53.9 \% \\
\text { Penicillin V 85.8\% }(\mathrm{P}<0.0001)\end{array}$ \\
\hline $\begin{array}{l}\text { Still, } \\
\quad 1994(23)\end{array}$ & $\begin{array}{l}\text { Paediatric (three to } 12 \text { years old) } \\
\text { Group A streptococcal } \\
\text { pharyngitis }(n=342)\end{array}$ & $\begin{array}{l}\text { Azithromycin } 12 \mathrm{mg} / \mathrm{kg} \text { by mouth daily for five days } \\
\text { Penicillin V } 250 \mathrm{mg} \text { by mouth tid for } 10 \text { days }\end{array}$ & $\begin{array}{l}\text { Clinical response } \\
\text { Azithromycin 95\% } \\
\text { Penicillin V 78.5\% (P<0.001) } \\
\text { Bacterial eradication } \\
\text { Azithromycin } 79.3 \% \\
\text { Penicillin V } 67.2 \%(P=0.035)\end{array}$ \\
\hline $\begin{array}{l}\text { Calhoun and } \\
\text { Hokanson, } \\
1993(25)\end{array}$ & $\begin{array}{l}\text { Adult } \\
\text { Acute maxillary sinusitis }(n=142)\end{array}$ & $\begin{array}{l}\text { Clarithromycin } 500 \mathrm{mg} \text { by mouth bid for seven } \\
\text { to } 14 \text { days } \\
\text { Amoxicillin } 500 \mathrm{mg} \text { by mouth tid for seven to } 14 \text { days }\end{array}$ & $\begin{array}{l}\text { Clinical response } \\
\text { Clarithromycin 91\% } \\
\text { Amoxicillin } 89 \% \text { (NS) }\end{array}$ \\
\hline $\begin{array}{c}\text { Dubois et al, } \\
1993 \text { (26) }\end{array}$ & $\begin{array}{l}\text { Adult } \\
\text { Acute maxillary sinusitis }(n=497)\end{array}$ & $\begin{array}{l}\text { Clarithromycin } 500 \mathrm{mg} \text { by mouth bid } \\
\text { Amoxicillin/clavulanate } 500 \mathrm{mg} \text { by mouth tid }\end{array}$ & $\begin{array}{l}\text { Clinical response } \\
\text { Clarithromycin 97\% } \\
\text { Amoxicillin/clavulanate 93\% (NS) } \\
\text { Bacterial eradication } \\
\text { Clarithromycin 87\% } \\
\text { Amoxicillin/clavulanate 90\% (NS) }\end{array}$ \\
\hline $\begin{array}{l}\text { Clement and } \\
\text { de Gandt, } \\
1998(28)\end{array}$ & $\begin{array}{l}\text { Adult } \\
\text { Acute maxillary or ethmoidal } \\
\quad \text { sinusitis (nonsevere) }(n=254)\end{array}$ & $\begin{array}{l}\text { Azithromycin } 500 \text { mg by mouth daily for three days } \\
\text { Amoxicillin/clavulanate } 500 \mathrm{mg} \text { by mouth tid } \\
\text { for } 10 \text { days }\end{array}$ & $\begin{array}{l}\text { Clinical response } \\
\text { Azithromycin } 87.5 \% \\
\text { Amoxicillin/clavulanate } \\
\text { 83.7\% (NS) }\end{array}$ \\
\hline $\begin{array}{l}\text { Principi, } \\
1995(29)\end{array}$ & $\begin{array}{l}\text { Paediatric } \\
\text { Acute otitis media }(n=484)\end{array}$ & $\begin{array}{l}\text { Azithromycin } 10 \mathrm{mg} / \mathrm{kg} \text { by mouth once daily } \\
\text { for three days } \\
\text { Amoxicillin/clavulanate } 13.3 \mathrm{mg} / \mathrm{kg} \text { by mouth tid } \\
\text { for } 10 \text { days }\end{array}$ & $\begin{array}{l}\text { Clinical response } \\
\text { Azithromycin } 92.6 \% \\
\text { Amoxicillin/clavulanate } \\
\text { 93.9\% (NS) }\end{array}$ \\
\hline
\end{tabular}


TABLE 2 (continued)

Comparative clinical trials: Respiratory tract infections

\begin{tabular}{rlr}
\hline Reference & Patient population & Agents compared \\
\hline $\begin{aligned} \text { Dagan et al, } \\
2000(30)\end{aligned}$ & $\begin{array}{l}\text { Paediatric } \\
\text { Acute otitis media }(\mathrm{n}=238)\end{array}$ & $\begin{array}{c}\text { Azithromycin } 10 \mathrm{mg} / \mathrm{kg} \text { by mouth on day } 1 \text {, then } \\
5 \mathrm{mg} / \mathrm{kg} \text { by mouth once daily on days } 2 \text { to } 5 \\
\text { Amoxicillin/clavulanate } 45 / 6.4 \mathrm{mg} / \mathrm{kg} \text { by mouth in } \\
\text { two divided doses for } 10 \text { days }\end{array}$ \\
$\begin{aligned} \text { Dagan et al, } \\
2000(31)\end{aligned}$ & $\begin{array}{l}\text { Paediatric } \\
\text { Acute otitis media }(\mathrm{n}=122)\end{array}$ & $\begin{array}{c}\text { Azithromycin } 10 \mathrm{mg} / \mathrm{kg} \text { by mouth once daily } \\
\text { for three days } \\
\text { Cefaclor } 40 \mathrm{mg} / \mathrm{kg} \text { by mouth in three divided doses } \\
\text { for } 10 \text { days }\end{array}$ \\
&
\end{tabular}

Pukander,

$1993(32)$

Coles et al, 1993 (33)

Aspin et al, 1994 (34)

McCarty et al, 1993 (35)

Gooch et al 1993 (36)

Wettengel et al, 1993 (37)

Adam, 1993 (38)
Paediatric

Acute otitis media $(n=79)$

Paediatric

Acute otitis media $(n=259)$

Paediatric

Acute otitis media $(n=180)$

Paediatric

Acute otitis media $(n=338)$

Paediatric

Acute otitis media $(n=379)$

Adult

Acute bronchitis of bacterial etiology Clarithromycin $250 \mathrm{mg}$ by mouth bid for seven days (mild to moderate) $(n=207)$

Adult

Purulent bronchitis $(n=221)$
Clarithromycin $7.5 \mathrm{mg} / \mathrm{kg}$ (maximum $500 \mathrm{mg}$ )

by mouth bid for seven to 10 days

Amoxicillin 20 mg/kg (maximum 750 mg) by mouth bid for seven to 10 days

Clarithromycin

Weight <25 kg: $125 \mathrm{mg}$ by mouth bid

Weight $>25 \mathrm{~kg}: 250 \mathrm{mg}$ by mouth bid for five days Amoxicillin

Weight <25 kg: $125 \mathrm{mg}$ by mouth tid

Weight $>25 \mathrm{~kg}: 250 \mathrm{mg}$ by mouth tid for five days

Clarithromycin $15 \mathrm{mg} / \mathrm{kg} /$ day by mouth in two divided doses for 10 days

Amoxicillin/clavulanate $40 \mathrm{mg} / \mathrm{kg} /$ day by mouth in three divided doses for 10 days

Clarithromycin $7.5 \mathrm{mg} / \mathrm{kg}$ (maximum $500 \mathrm{mg}$ )

by mouth bid for 10 days

Amoxicillin/clavulanate 13.3 mg/kg (maximum 500 mg) by mouth tid for 10 days

Clarithromycin $7.5 \mathrm{mg} / \mathrm{kg}$ (maximum $500 \mathrm{mg}$ )

by mouth bid for 10 days

Cefaclor $20 \mathrm{mg} / \mathrm{kg}$ (maximum $500 \mathrm{mg}$ ) by mouth bid for 10 days maximum

Cefaclor $500 \mathrm{mg}$ by mouth tid for seven days maximum

Clarithromycin $250 \mathrm{mg}$ by mouth bid for five days Clarithromycin $250 \mathrm{mg}$ by mouth bid for 10 days
Results

Clinical response in culture-positive patients:

Azithromycin $70 \%$

Amoxicillin/clavulanate 86\% $(\mathrm{P}=0.023)$

Clinical response

Azithromycin $82 \%$

Cefaclor 85\% (NS)

Bacteriological response

Haemophilus influenzae Azithromycin 53\% Cefaclor 52\% (NS)

Streptococcus pneumoniae Azithromycin 29\%

Cefaclor 37\% (NS)

Clinical response

Clarithromycin 93\%

Amoxicillin 90\% (NS)

Clinical response

Clarithromycin 96\%

Amoxicillin 96\% (NS)

Clinical response

Clarithromycin 93\%

Amoxicillin/clavulanate 95\% (NS)

Clinical response

Clarithromycin 90\%

Amoxicillin/clavulanate 92\% (NS)

Clinical response

Clarithromycin 86\%

Cefaclor 90\% (NS)

Clinical response Clarithromycin 99.5\%

Cefaclor $97.9 \%$ (NS)

Bacteriological cure

Clarithromycin $94.6 \%$

Cefaclor 90.3\% (NS)

Clinical response

Clarithromycin (five days) 98\%

Clarithromycin (10 days) 99\% (NS)

Bacteriological cure Clarithromycin (five days) 100\%

Clarithromycin (10 days)

94\% (NS)

Continued on next page 
TABLE 2 (continued)

Comparative clinical trials: Respiratory tract infections

\begin{tabular}{|c|c|c|}
\hline Reference & Patient population & Agents compared \\
\hline $\begin{array}{l}\text { Neu and Chick, } \\
1993 \text { (41) }\end{array}$ & $\begin{array}{l}\text { Adult } \\
\text { Community-acquired lower } \\
\text { respiratory tract infections } \\
\text { (bacterial pneumonia, acute } \\
\text { bacterial exacerbations of } \\
\text { chronic bronchitis or asthmatic } \\
\text { bronchitis) }(n=213)\end{array}$ & $\begin{array}{l}\text { Clarithromycin } 500 \mathrm{mg} \text { by mouth bid for seven } \\
\text { to } 14 \text { days } \\
\text { Cefixime } 400 \mathrm{mg} \text { by mouth daily for seven } \\
\text { to } 14 \text { days }\end{array}$ \\
\hline $\begin{array}{l}\text { Fong et al, } \\
1995(42)\end{array}$ & $\begin{array}{l}\text { Adult } \\
\text { Lower respiratory tract infections } \\
\text { (acute bronchitis or exacerbation } \\
\text { of chronic bronchitis, or } \\
\text { pneumonia) }(n=95)\end{array}$ & $\begin{array}{l}\text { Clarithromycin } 250 \text { or } 500 \text { mg by mouth bid for } \\
\text { seven to } 14 \text { days } \\
\text { Cefaclor } 250 \text { or } 500 \mathrm{mg} \text { by mouth tid for seven } \\
\text { to } 14 \text { days }\end{array}$ \\
\hline $\begin{array}{l}\text { Chodosh et al, } \\
1998 \text { (39) }\end{array}$ & $\begin{array}{l}\text { Adult } \\
\text { Acute bacterial exacerbations of } \\
\text { chronic bronchitis }(n=234)\end{array}$ & $\begin{array}{l}\text { Clarithromycin } 500 \mathrm{mg} \text { by mouth bid for } 14 \text { days } \\
\text { Ciprofloxacin } 500 \mathrm{mg} \text { by mouth bid for } 14 \text { days }\end{array}$ \\
\hline $\begin{array}{l}\text { Anzueto et al, } \\
1998(40)\end{array}$ & $\begin{array}{l}\text { Adult } \\
\text { Complicated and/or severe } \\
\text { acute exacerbations of chronic } \\
\text { bronchitis }(n=2180)\end{array}$ & $\begin{array}{l}\text { Clarithromycin } 500 \mathrm{mg} \text { by mouth bid for } 10 \text { days } \\
\text { Ciprofloxacin } 750 \mathrm{mg} \text { by mouth bid for } 10 \text { days }\end{array}$ \\
\hline $\begin{array}{l}\text { Bradbury, } \\
1993 \text { (43) }\end{array}$ & $\begin{array}{l}\text { Adult } \\
\text { Lower respiratory tract infections } \\
\text { (acute bronchitis, acute infective } \\
\text { exacerbations of chronic } \\
\text { bronchitis or pneumonia) }(n=510)\end{array}$ & $\begin{array}{l}\text { Azithromycin } 500 \mathrm{mg} \text { by mouth daily for three days } \\
\text { Clarithromycin } 250 \mathrm{mg} \text { by mouth bid for } 10 \text { days } \\
\text { ) }\end{array}$ \\
\hline
\end{tabular}

Biebuyck, $1996(44)$

Jang et al, 1995 (47)

Chien et al, $1993(48)$

Rizzato et al, 1995 (49)
Adult

Acute tracheobronchitis or acute infectious exacerbations of chronic bronchitis $(n=759)$

Adult

Community-acquired pneumonia $(n=40)$

Adult

Community-acquired pneumonia $(n=268)$
Azithromycin $500 \mathrm{mg}$ by mouth daily for three days Amoxicillin/clavulanate $625 \mathrm{mg}$ by mouth tid for five to 10 days

Clarithromycin $250 \mathrm{mg}$ by mouth bid for 14 days

Erythromycin $500 \mathrm{mg}$ by mouth four times daily for 14 days

Clarithromycin $250 \mathrm{mg}$ by mouth bid for seven to 14 days

Erythromycin stearate $500 \mathrm{mg}$ by mouth four times daily for seven to 14 days

Adult

Community-acquired pneumonia

Azithromycin $500 \mathrm{mg}$ by mouth daily for three days (low to moderately severe) $(n=40)$
Clarithromycin $250 \mathrm{mg}$ by mouth bid for eight to
Results

Clinical response

Clarithromycin 86\%

Cefixime $88 \%$ (NS)

Bacteriological eradication

Clarithromycin 91\%

Cefixime 90\% (NS)

Clinical response

Clarithromycin 94.7\%

Cefaclor 90.2\% (NS)

Bacteriological eradication

Clarithromycin $72.2 \%$

Cefaclor $70 \%$ (NS)

Clinical response

Clarithromycin 82\%

Ciprofloxacin 90\% (NS)

Bacteriological eradication

Clarithromycin 77\%

Ciprofloxacin 91\% ( $\mathrm{P}=0.01)$

Clinical response

Clarithromycin 90\%

Ciprofloxacin 93\% (NS)

Bacteriological eradication

Clarithromycin 96\%

Ciprofloxacin 96\% (NS)

Clinical response

Azithromycin 97\%

Clarithromycin $100 \%$ (NS)

Bacteriological response

Azithromycin 100\%

Clarithromycin 95\% (NS)

Clinical response

Azithromycin 89.7\%

Amoxicillin/clavulanate $80.2 \%$ $(P=0.0003)$

Clinical response

Clarithromycin 95\%

Erythromycin 90\% (NS)

Clinical response

Clarithromycin 97\%

Erythromycin 96\% (NS)

Bacteriological response

Clarithromycin 88\%

Erythromycin 100\% (NS)

Clinical response

Azithromycin 20\%

Clarithromycin 17\% (NS)

Bacteriological response

Azithromycin 100\%

Clarithromycin 100\% (NS) 
TABLE 2 (continued)

Comparative clinical trials: Respiratory tract infections

\begin{tabular}{|c|c|c|c|}
\hline Reference & Patient Population & Agents Compared & Results \\
\hline $\begin{array}{l}\text { Bohte et al, } \\
1995 \text { (50) }\end{array}$ & $\begin{array}{l}\text { Adult } \\
\text { Community-acquired pneumonia } \\
\text { (hospitalized) }(n=108)\end{array}$ & $\begin{array}{l}\text { Azithromycin } 500 \mathrm{mg} \text { by mouth bid for one day, } \\
\text { then } 500 \mathrm{mg} \text { by mouth daily for } 4 \text { days, } \\
\text { versus } \\
\text { Benzylpenicillin } 1 \mathrm{MU} \text { intravenously four times daily } \\
\text { until afebrile for five days, or } \\
\text { Erythromycin } 500 \mathrm{mg} \text { by mouth four times daily } \\
\text { for } 10 \text { days }\end{array}$ & $\begin{array}{l}\text { Clinical response } \\
\text { Pneumococcal } \\
\text { Azithromycin } 83 \% \\
\text { Benzylpenicillin } 66 \% \text { (NS) } \\
\text { Nonpneumococcal } \\
\text { Azithromycin } 79 \% \\
\text { Erythromycin } 76 \% \text { (NS) }\end{array}$ \\
\hline $\begin{array}{l}\text { Vergis et al, } \\
2000(51)\end{array}$ & $\begin{array}{l}\text { Adult } \\
\text { Community-acquired pneumonia } \\
\text { (hospitalized) }(n=145)\end{array}$ & $\begin{array}{l}\text { Azithromycin } 500 \mathrm{mg} \text { intravenously daily for two to } \\
\text { five days, then } 500 \mathrm{mg} \text { by mouth daily to complete } \\
\text { seven to } 10 \text { days, versus } \\
\text { [Cefuroxime } 750 \mathrm{mg} \text { intravenously every } 8 \mathrm{~h} \text { for two to } \\
\text { seven days, then cefuroxime axetil } 500 \mathrm{mg} \text { by mouth } \\
\text { bid to complete seven to } 10 \text { days, and } \\
\text { Erythromycin } 500 \text { to } 1000 \mathrm{mg} \text { intravenously by mouth } \\
\text { every } 6 \mathrm{~h} \text { for up to } 21 \text { days] }\end{array}$ & $\begin{array}{l}\text { Clinical response } \\
\text { Azithromycin } 91 \% \\
\text { Cefuroxime and erythromycin } \\
91 \% \text { (NS) }\end{array}$ \\
\hline $\begin{array}{l}\text { Plouffe et al, } \\
2000(52)\end{array}$ & $\begin{array}{l}\text { Adult } \\
\text { Community-acquired pneumonia } \\
\text { (hospitalized) }(n=403)\end{array}$ & $\begin{array}{l}\text { Azithromycin } 500 \mathrm{mg} \text { intravenously daily for two to } \\
\text { five days then } 500 \mathrm{mg} \text { by mouth daily to complete } \\
\text { seven to } 10 \text { days, versus } \\
\text { [Cefuroxime } 750 \mathrm{mg} \text { intravenously every } 8 \mathrm{~h} \text { for two to } \\
\text { seven days, then cefuroxime axetil } 500 \mathrm{mg} \text { by mouth } \\
\text { bid to complete seven to } 10 \text { days with or without } \\
\text { Erythromycin } 500 \text { by mouth or } 500 \text { to } 1000 \mathrm{mg} \\
\text { intravenously every } 6 \text { h for up to } 21 \text { days] }\end{array}$ & $\begin{array}{l}\text { Clinical response } \\
\text { Azithromycin } 77 \% \\
\text { Cefuroxime with or without } \\
\text { erythromycin } 74 \% \text { (NS) } \\
\text { Bacterial eradication } \\
\text { Haemophilus influenzae } \\
\text { Azithromycin } 94 \% \\
\text { Cefuroxime with or without } \\
\text { erythromycin } 73 \%(\mathrm{NS})\end{array}$ \\
\hline $\begin{array}{l}\text { Uzun et al, } \\
1994 \text { (53) }\end{array}$ & $\begin{array}{l}\text { Adult } \\
\text { Community-acquired } \\
\text { pneumococcal pneumonia }(n=25)\end{array}$ & $\begin{array}{l}\text { Azithromycin } 1000 \text { mg by mouth on first day, then } \\
500 \text { mg by mouth daily on following two days }\end{array}$ & $\begin{array}{l}\text { Clinical response } 96 \% \\
\text { Bacterial response } 93 \%\end{array}$ \\
\hline
\end{tabular}

NS Not significant

cal success and overall bacteriological response rates in patients treated with oral clarithromycin $500 \mathrm{mg}$ or ciprofloxacin $750 \mathrm{mg}$ twice daily (40). Compared with cefixime as a seven- to 14-day outpatient treatment, both therapies were found to be effective for the treatment of mild to moderate pneumonia and acute bacterial exacerbations of chronic bronchitis caused by $H$ influenzae, $M$ catarrhalis or $S$ pneumoniae in adults (41). Comparison of clarithromycin with cefaclor for the same indication showed similar favourable clinical response and bacteriological eradication rates in both groups (42). An open-label study employing clarithromycin $(250 \mathrm{mg}$ orally twice daily for 10 days) or azithromycin (500 mg orally once daily for three days) for the treatment of lower respiratory tract infections, including acute bronchitis, acute infective exacerbations of chronic bronchitis and pneumonia, showed similar effectiveness for both drugs (43). Azithromycin has also been shown to have a statistically significant higher overall clinical response rate than amoxicillin/clavulanate for the treatment of acute tracheobronchitis or acute infectious exacerbations of chronic bronchitis (44).

Pertussis: Only two clinical trials have been published that have evaluated the use of either azithromycin or clarithromycin for the treatment of pertussis in children $(45,46)$. Small numbers of subjects in each trial make it difficult to recommend these agents as first-line therapy. It is likely that the newer agents are equivalent to standard therapy, such as erythromycin, for this infection.

Community-acquired pneumonia: The empirical treatment of community-acquired pneumonia (CAP) with the macrolides has been well studied. Clarithromycin $250 \mathrm{mg}$ orally bid has been shown to be just as effective as erythromycin $500 \mathrm{mg}$ orally four times daily as a seven- to 14-day therapy for this condition in adults $(47,48)$. Oral azithromycin has also compared favourably with oral clarithromycin (49) and oral erythromycin (50) for the treatment of CAP. More recently, azithromycin (500 mg intravenously once daily for two days followed by $500 \mathrm{mg}$ orally once daily; total duration of seven to 10 days) was compared with the combination of cefuroxime (750 mg intravenously every $8 \mathrm{~h}$ for two to seven days followed by $500 \mathrm{mg}$ orally twice daily; total duration seven to 10 days) and erythromycin (500 to $1000 \mathrm{mg}$ intravenously or orally every $6 \mathrm{~h}$; total duration up to 21 days) in hospitalized patients (51). Clinical cure rates were similar ( $91 \%$ in each group, $\mathrm{P}=0.95$ ). Another openlabel trial comparing intravenous-to-oral azithromycin therapy with cefuroxime therapy with the addition of erythromycin therapy, if deemed necessary by the clinician, has been published (52). Clinical cure or improvement was noted in $77 \%$ of 
TABLE 3

Frequency of gastrointestinal adverse effects of macrolides at recommended doses

\begin{tabular}{lccc}
\hline $\begin{array}{l}\text { Adverse } \\
\text { effect }\end{array}$ & $\begin{array}{c}\text { Erythromycin } \\
(\mathbf{\%})^{*}\end{array}$ & $\begin{array}{c}\text { Clarithromycin } \\
\mathbf{( \% )}\end{array}$ & $\begin{array}{c}\text { Azithromycin } \\
\mathbf{( \% )}\end{array}$ \\
\hline Diarrhea & 5.3 & 3 & 4.3 \\
Dyspepsia & 5 to $7^{\S}$ & 2 & $<1$ \\
Abdominal & 6.9 & 2 & 2.6 \\
$\quad$ pain & & & 1.3 \\
Vomiting & 2.9 & 1 & 3.5 \\
Nausea & 5.5 & 4 & \\
\hline
\end{tabular}

Data from references $* 87,{ }^{\dagger} 95,{ }^{\ddagger} 14,{ }^{\S} 12$

the azithromycin group and $74 \%$ of the cefuroxime with or without erythromycin group (not significant). Until further information is available, the treatment of patients with bacteremic $S$ pneumoniae pneumonia with azithromycin monotherapy should be undertaken with caution due to low serum concentrations relative to beta-lactam agents. An open-label study in 25 patients using a three-day course of the azalide reported that a patient with $S$ pneumoniae bacteremia and pneumonia failed to respond to antibiotic therapy and died in respiratory failure (53).

The effectiveness of azithromycin for CAP has also been studied in children. Clinical response was similar when azithromycin was compared with amoxicillin/clavulanate (age five years or younger) or erythromycin estolate (age older than five years) (90.6\% and $87.1 \%$, respectively) (54). Chlamydia pneumoniae and $M$ pneumoniae eradication was at least as successful with azithromycin as with the comparator antimicrobial ( $81 \%$ and $100 \%$ for azithromycin versus $100 \%$ and $57 \%$ for amoxicillin/clavulanate, respectively).

Skin and skin structure infections: The efficacy of clarithromycin in the treatment of mild to moderate skin and skin structure infections has been assessed in adults and children. Clarithromycin (500 mg twice daily for five to 14 days) produces clinical response rates comparable with erythromycin (250 mg four times daily for less than 14 days) and cefadroxil (500 mg twice daily for five to 14 days) (55). Similarly, children treated with clarithromycin $7.5 \mathrm{mg} / \mathrm{kg}$ (maximum $500 \mathrm{mg}$ ) twice daily responded equally well to cefadroxil $15 \mathrm{mg} / \mathrm{kg}$ (maximum $1000 \mathrm{mg}$ ) twice daily (56).

Azithromycin $(500 \mathrm{mg}$ orally on the first day followed by $250 \mathrm{mg}$ for four more days) has been shown to be as effective as oral erythromycin (500 mg four times daily for seven days) $(57,58)$, cephalexin (500 mg twice daily for 10 days) $(59,60)$ and cloxacillin (500 mg twice daily for seven days) (58) in the treatment of mild to moderate acute bacterial infections of skin or soft tissue in adults. In children aged six months to 12 years, therapy with azithromycin $10 \mathrm{mg} / \mathrm{kg}$ for three days was shown to be as effective as cefaclor $20 \mathrm{mg} / \mathrm{kg} /$ day in three divided doses for the treatment of mild to moderate dermatological conditions and abscesses (61).

Sexually transmitted diseases: Several clinical trials have demonstrated the effectiveness of azithromycin for the treatment of sexually transmitted chlamydial infections. A single
$1000 \mathrm{mg}$ dose of oral azithromycin was shown to be effective and well tolerated compared with doxycycline $100 \mathrm{mg}$ orally twice daily for seven days in men treated for either uncomplicated gonococcal urethritis and/or urethritis caused by $C$ trachomatis and Ureaplasma urealyticum (62). Similarly, other trials have shown chlamydial cervicitis and nongonococcal urethritis to respond equally well to either azithromycin or doxycycline $(63,64)$ and clarithromycin $250 \mathrm{mg}$ orally twice daily for seven days or doxycycline (65). However, in the latter trial, doxycycline was more effective than clarithromycin in eradicating $U$ urealyticum. Treatment of chlamydial infection in pregnant women and their sexual partners with single-dose azithromycin has been shown to be superior to therapy with standard course erythromycin for women, combined with tetracycline for their sexual partners. The proportion of positive cultures for $C$ trachomatis at four weeks was significantly lower in women and partners randomized to single dose azithromycin (4.5\%) than in those randomized to standard dose erythromycin or tetracycline (21.1\%) (66). In this same study, adverse effects were significantly less in the azithromycin group (7.4\%) compared with the group receiving erythromycin $(38.8 \%)$ or tetracycline (28.6\%).

Nontuberculous mycobacterial infections - MAC prophylaxis and therapy: Clarithromycin and azithromycin have been studied in the prophylaxis and treatment of HIV-infected patients with MAC infections. Clarithromycin reduced the incidence of disseminated infection by $10 \%$ and improved survival (hazard ratio $0.75, \mathrm{P}=0.026$ ) in patients with advanced AIDS compared with a placebo (67). Clarithromycin when used as a monotherapy has also been shown to be as effective as when used in combination with rifabutin for primary prophylaxis in HIV-infected patients (68). A comparison of azithromycin (1200 mg once weekly) alone, rifabutin (300 mg daily) alone or both for MAC prophylaxis showed that the combination produced the lowest cumulative incidence of disseminated MAC infection (2.8\%) at one year of prophylactic therapy (69).

Clarithromycin in doses of 500 to $1000 \mathrm{mg}$ twice daily has been demonstrated to eradicate MAC bacteremia and improve symptomatology $(70,71)$. In disseminated MAC, however, macrolide monotherapy can lead to drug resistance (72). Therefore, the use of combination therapy is advocated (73). The addition of ethambutol to macrolide-containing regimens for MAC bacteremia has been shown to decrease the emergence of resistance (74). A clarithromycin-based threedrug regimen was superior to a four-drug regimen without clarithromycin in resolving MAC bacteremia and increasing survival rates in patients with AIDS (75). Most recently, a trial demonstrated that a combination of clarithromycin and ethambutol was superior to azithromycin and ethambutol for the treatment of MAC bacteremia in HIV-infected patients (76). Median time to clearance of bacteremia was significantly faster in the clarithromycin group (4.38 weeks versus longer than 16 weeks in the azithromycin group). Bacteremia resolved in a greater proportion of patients randomized to the clarithromycin group at 16 weeks (85.7\% versus $37.5 \%)$. 
TABLE 4

Potential clinically significant drug interactions involving erythromycin, clarithromycin and azithromycin

\begin{tabular}{|c|c|c|c|}
\hline \multirow[b]{2}{*}{ Interacting drug } & \multicolumn{3}{|c|}{ Effect when administered with macrolide indicated } \\
\hline & Erythromycin & Clarithromycin & Azithromycin \\
\hline $\begin{array}{l}\text { Theophylline and } \\
\text { related drugs }\end{array}$ & $\begin{array}{l}\text { Increased serum theophylline level } \\
\text { with possible toxicity }\end{array}$ & $\begin{array}{l}\text { Increased serum theophylline level } \\
\text { with possible toxicity }\end{array}$ & $\begin{array}{l}\text { No interaction observed } \\
\text { but caution advised }\end{array}$ \\
\hline Carbamazepine & $\begin{array}{l}\text { Increased serum carbamazepine level } \\
\text { with possible toxicity }\end{array}$ & $\begin{array}{l}\text { Increased serum carbamazepine level } \\
\text { with possible toxicity }\end{array}$ & No interaction reported \\
\hline $\begin{array}{l}\text { Cisapride (no longer } \\
\text { marketed in Canada) }\end{array}$ & $\begin{array}{l}\text { Increased serum cisapride level with possible } \\
\text { cardiotoxicity (QT prolongation) }\end{array}$ & $\begin{array}{l}\text { Increased serum cisapride level with } \\
\text { possible cardiotoxicity (QT prolongation) }\end{array}$ & No interaction reported \\
\hline Cyclosporine, tacrolimus & $\begin{array}{l}\text { Increased serum immunosuppressant level } \\
\text { with possible toxicity }\end{array}$ & $\begin{array}{l}\text { Increased serum immunosuppressant } \\
\text { level with possible toxicity }\end{array}$ & No interaction reported \\
\hline $\begin{array}{l}\text { Astemizole, terfenadine } \\
\text { (no longer marketed in } \\
\text { Canada) }\end{array}$ & $\begin{array}{l}\text { Increased serum level of antihistamine } \\
\text { leading to possible cardiotoxicity } \\
\text { (QT prolongation) }\end{array}$ & $\begin{array}{l}\text { Increased level on antihistamine } \\
\text { leading to possible cardiotoxicity } \\
\text { (QT prolongation) }\end{array}$ & No interaction reported \\
\hline $\begin{array}{l}\text { Alprazolam, diazepam, } \\
\text { midazolam, triazolam }\end{array}$ & $\begin{array}{l}\text { Increased serum level of benzodiazepines } \\
\text { that undergo oxidative metabolism with } \\
\text { possible toxicity }\end{array}$ & No interaction reported & No interaction reported \\
\hline Buspirone & $\begin{array}{l}\text { Increased serum buspirone level with } \\
\text { possible toxicity }\end{array}$ & $\begin{array}{l}\text { No interaction observed but } \\
\text { caution advised }\end{array}$ & No interaction reported \\
\hline $\begin{array}{l}\text { Ergot alkaloids } \\
\text { (including bromocriptine) }\end{array}$ & Increased risk of peripheral ischemia & Increased risk of peripheral ischemia & No interaction reported \\
\hline Lovastatin & $\begin{array}{l}\text { Possible severe myopathy or rhabdomyolysis; } \\
\text { caution advised with all 3-hydroxy-3-methyl- } \\
\text { glutaryl coenzyme A reductase inhibitors }\end{array}$ & $\begin{array}{l}\text { No interaction observed but } \\
\text { caution advised }\end{array}$ & $\begin{array}{l}\text { No interaction observed } \\
\text { but caution advised }\end{array}$ \\
\hline Rifabutin, rifampin & Possible decrease in antimicrobial effects & Possible decrease in antimicrobial effects & No interaction reported \\
\hline Disopyramide & $\begin{array}{l}\text { Increased serum disopyramide level } \\
\text { with possible toxicity }\end{array}$ & $\begin{array}{l}\text { Increased serum disopyramide level } \\
\text { with possible toxicity }\end{array}$ & No interaction reported \\
\hline Digoxin & $\begin{array}{l}\text { Increased serum digoxin level with } \\
\text { possible toxicity }\end{array}$ & $\begin{array}{l}\text { Increased serum digoxin level with } \\
\text { possible toxicity }\end{array}$ & No interaction reported \\
\hline Warfarin & $\begin{array}{l}\text { Reduction in warfarin clearance leading to } \\
\text { potentiation of anticoagulant effect }\end{array}$ & $\begin{array}{l}\text { Reduction in warfarin clearance leading } \\
\text { to potentiation of anticoagulant effect }\end{array}$ & No interaction reported \\
\hline
\end{tabular}

Data from reference 96

However, it is possible that, in this study, the dose of azithromycin (600 mg once daily) studied was too low.

Infections with other nontuberculous mycobacteria: Clarithromycin therapy in combination with other agents may be effective for the treatment of nontuberculous, nonMAC mycobacterial infections. If required due to protease inhibitor drug interactions, clarithromycin or rifabutin may be added to isoniazid and ethambutol instead of rifampin for the treatment of $M$ kansasii pulmonary disease. This recommendation is based on the excellent in vitro susceptibility of $M$ kansasii to clarithromycin (77). Excellent in vitro susceptibility to clarithromycin has also led to this agent being recommended as one of the treatments for cutaneous infections with $M$ marinum, for pulmonary infections with $M$ xenopi and for cutaneous or pulmonary infections with Mycobacterium abscessus or $M$ fortuitum (77). Clarithromycin is also recommended for the treatment of cervical lymphadenitis caused by nontuberculous mycobacteria in patients with extensive disease or poor response to surgical excision (77). Long term clarithromycin monotherapy has been shown to be effective for the treatment of cutaneous infection with $M$ chelonae in an open-label, noncomparative trial (78). Promising results were also observed in a small trial assessing improvement in clinical and laboratory parameters; patients with previously untreated Mycobacterium leprae infection received clarithromycin monotherapy (79).
Peptic ulcer disease and $\boldsymbol{H}$ pylori infection: The association of $H$ pylori with peptic ulcer disease is now well established. There have been six published meta-analyses on regimens for $H$ pylori eradication (80-85). All have shown that three drug regimens using clarithromycin and a proton pump inhibitor in combination with amoxicillin or metronidazole have eradication rates between $80 \%$ to $90 \%$ assessed by intention-to-treat analysis. In one meta-analysis examining the role of high versus low dose clarithromycin administered with a proton pump inhibitor and amoxicillin or metronidazole, the best treatment results by intention-totreat analysis were seen with $500 \mathrm{mg}$ twice daily compared with $250 \mathrm{mg}$ twice daily (amoxicillin, 86.6\% versus $78.2 \%$; metronidazole, $88.3 \%$ versus $86.7 \%$ ) (82). Azithromycin for $H$ pylori infection has not been as well studied as clarithromycin and is not currently recommended.

\section{ADVERSE EFFECTS}

Erythromycin is one of the safest antibiotics in clinical use. Gastrointestinal upset is the most common adverse event associated with macrolide therapy and is dose-related (Table 3). Macrolides act as gastric prokinetic agents through stimulation of motilin receptors in the gastrointestinal tract. It is believed that erythromycin's high affinity for these receptors, relative to that of clarithromycin and azithromycin, results in the higher incidence of gastrointestinal upset observed during 
TABLE 5

Oral dosing of select macrolides and azalides

\begin{tabular}{|c|c|c|c|}
\hline & Erythromycin base & Clarithromycin & Azithromycin \\
\hline Dose (adult) & $\begin{array}{l}250 \text { to } 500 \text { mg by mouth } \\
\text { four times daily for } 10 \text { days }\end{array}$ & $\begin{array}{l}250 \text { to } 500 \text { mg by mouth bid } \\
\text { for } 10 \text { days }\end{array}$ & $\begin{array}{l}500 \mathrm{mg} \text { by mouth on first day } \\
\text { followed by } 250 \mathrm{mg} \text { by mouth daily } \\
\text { on the following four days }\end{array}$ \\
\hline Cost per course (adult)* & $\$ 1.81$ to $\$ 3.62$ & $\$ 29.58$ to $\$ 59.16$ & $\$ 29.60$ \\
\hline $\begin{array}{l}\text { Dosing in renal and/or } \\
\text { hepatic failure }\end{array}$ & $\begin{array}{l}\text { No adjustment in renal impairment; } \\
\text { use with caution if there is } \\
\text { hepatic impairment }\end{array}$ & $\begin{array}{l}\text { Reduce dose and/or frequency } \\
\text { in renal impairment } \\
\text { (creatinine clearance }<0.5 \mathrm{~mL} / \mathrm{s} \text { ) }\end{array}$ & $\begin{array}{l}\text { No dose adjustment if there is mild to } \\
\text { moderate renal or hepatic impairment; } \\
\text { use with caution if creatinine clearance } \\
<0.5 \mathrm{~mL} / \mathrm{s} \text {; avoid if there is severe } \\
\text { hepatic disease }\end{array}$ \\
\hline Administration & $\begin{array}{l}1 \mathrm{~h} \text { before or } 2 \mathrm{~h} \text { after food or antacids; } \\
\text { stability in presence of acid varies } \\
\text { with formulation administered }\end{array}$ & With or without food & $\begin{array}{l}\text { Capsules and suspension: } 1 \mathrm{~h} \text { before } \\
\text { or } 2 \mathrm{~h} \text { after food or antacids } \\
\text { Tablets (with or without food): }\end{array}$ \\
\hline Paediatric dosing & $\begin{array}{l}7.5 \text { to } 12.5 \mathrm{mg} / \mathrm{kg} / \mathrm{dose} \text { by mouth } \\
\text { administered four times daily }\end{array}$ & $\begin{array}{l}3.75 \text { to } 7.5 \mathrm{mg} / \mathrm{kg} / \text { dose by mouth } \\
\text { administered twice daily } \\
\text { (maximum } 1000 \mathrm{mg} / \text { day) }\end{array}$ & $\begin{array}{l}10 \mathrm{mg} / \mathrm{kg} / \text { day by mouth on the first day } \\
\text { followed by } 5 \mathrm{mg} / \mathrm{kg} / \text { day by mouth } \\
\text { administered as a single dose on the } \\
\text { following four days }(12 \mathrm{mg} / \mathrm{kg} / \text { day for } \\
\text { five days for pharyngitis/tonsillitis) }\end{array}$ \\
\hline
\end{tabular}

${ }^{*}$ Drug acquisition costs for solid oral dosage forms, from reference 97

TABLE 6

Parenteral dosing of erythromycin and azithromycin

\begin{tabular}{|c|c|c|}
\hline & Erythromycin lactobionate & Azithromycin dihydrate (reference 14) \\
\hline Dose (adult) & 500 to $1000 \mathrm{mg}$ intravenously every $6 \mathrm{~h}$ & $500 \mathrm{mg}$ intravenously every $24 \mathrm{~h}$ \\
\hline Cost per day (adult)* & $\$ 8.60$ to $\$ 17.20$ & $\$ 20.00$ \\
\hline $\begin{array}{l}\text { Dosing in renal and/or } \\
\text { hepatic failure }\end{array}$ & Use with caution in renal and hepatic impairment & $\begin{array}{l}\text { No dose adjustment if mild to moderate renal } \\
\text { or hepatic impairment; use with caution if creatinine } \\
\text { clearance }<0.5 \mathrm{~mL} / \mathrm{s} \text {; avoid if severe hepatic disease }\end{array}$ \\
\hline Administration & $\begin{array}{l}\text { Dilute each dose in } 250 \text { to } 500 \mathrm{~mL} \text { sodium chloride } \\
0.9 \% \text { to achieve concentration of } \leq 4 \mathrm{mg} / \mathrm{L} ; \\
\text { infuse each dose over at least } 30 \text { minutes }\end{array}$ & $\begin{array}{c}\text { Dilute each dose in } 250 \text { to } 500 \mathrm{~mL} \text { sodium chloride } \\
0.9 \% \text { or dextrose } 5 \% \text {; infuse each dose over } 1 \mathrm{~h}\end{array}$ \\
\hline Pediatric dosing & $\begin{array}{l}7.5 \text { to } 12.5 \mathrm{mg} / \mathrm{kg} / \text { dose by mouth } \\
\text { administered four times daily }\end{array}$ & Not approved for use \\
\hline
\end{tabular}

*Hospital drug acquisition costs

therapy with this macrolide (86). The incidence of significant gastrointestinal effects (ie, nausea, diarrhea, abdominal pain and vomiting) is reported to be $20 \%$ to $35 \%$ with erythromycin and $10 \%$ to $15 \%$ for clarithromycin or azithromycin (87). Venous irritation and phlebitis occur commonly with parenteral erythromycin therapy, and may be minimized with a reduction in the concentration and/or infusion rate (88). Parenteral azithromycin appears to be better tolerated with an incidence of infusion site reactions of $3 \%$ to $6 \%$ (14). Other adverse effects reported during clinical trials with a frequency of less than $2 \%$ include headache, hepatic dysfunction, changes in neutrophil or leukocyte counts, and skin rash. Taste perversion has been reported in patients receiving clarithromycin therapy at a frequency of $2 \%$. Bilateral, sensorineural ototoxicity has been reported with each of the macrolide derivatives. It is typically reversible with drug discontinuation and appears to be related to high serum concentrations arising from aggressive dosing, or hepatic or renal dysfunction $(13,89)$.

\section{DRUG INTERACTIONS}

Several pharmacokinetic drug interactions have been documented with erythromycin, an inhibitor of the cytochrome P450 (subset CYP3A) enzyme system through inactivation by the formation of an inactive complex (Table 4). The clearance of theophylline, carbamazepine, nonsedating antihistamines (ie, terfenadine, astemizole and, potentially, loratidine), ergot alkaloids, cyclosporine and warfarin may decrease if erythromycin is administered concomitantly. Clinically significant toxicity may ensue. Clarithromycin forms microsomal complexes to a lesser extent, and azithromycin does not appear to inactivate cytochrome $\mathrm{P} 450$ at all. Clarithromycin may cause clinically significant increases in carbamazepine, terfenadine and theophylline concentrations via inhibition of CYP3Amediated metabolism (2). Concomitant use should be avoided or monitored closely. Azithromycin appears to have no clinically significant pharmacokinetic effect on drugs metabolized by cytochrome P450 (90).

When physical complexes are formed with antacids or food, there may be a reduction in the bioavailability of erythromycin. Food and antacids do not appear to cause a clinically significant interaction when administered with clarithromycin. However, peak azithromycin serum levels may be reduced when administered as a capsule or oral suspension concomitantly with food or antacids (14). Therefore, this drug is recommended for administration on an empty stomach in the absence of antacids. 


\section{DOSING AND ADMINISTRATION}

The recommended dosages and recommendations regarding dosing adjustment in renal and/or hepatic failure are presented in Table 5. Estimated drug acquisition costs for total regimen are also provided. Only erythromycin and azithromycin are available in parenteral forms for intravenous administration. Parenteral azithromycin therapy may be a useful alternative to parenteral erythromycin when a reduction in fluid administration or frequency of administration is desired. A dosing comparison of these agents is provided in Table 6.

\section{CONCLUSIONS AND RECOMMENDATIONS}

The macrolide clarithromycin and the azalide azithromycin offer potential therapeutic and tolerability advantages over erythromycin. For the majority of patients treated (eg, those suffering from community-acquired respiratory tract infections), these drugs offer a more tolerable gastrointestinal adverse event profile and a simpler dosing regimen. The antimicrobial spectrum and clinical trial evidence support the use of either azithromycin or clarithromycin as first-line agents or alternatives to erythromycin in the treatment of CAP in the ambulatory setting. Both agents are acceptable for the treatment of hospitalized patients with CAP in combination with second- or third-generation cephalosporins. The introduction of azithromycin injection offers an alternative to parenteral erythromycin in this setting. The extended antimicrobial coverage of clarithromycin versus MAC and other nontuberculous mycobacteria is clinically relevant. For peptic ulcer disease caused by $H$ pylori, triple-drug regimens containing clarithromycin are considered first-line therapy. In the treatment of uncomplicated genital tract chlamydial infection and nongonococcal urethritis, single dose azithromycin is now the standard. For the treatment of skin and skin structure infections, more effective and less expensive agents exist, rendering these agents to second-line status.

The evidence cited in these recommendations has been classified into five levels, which have been used in the development of other clinical practice guidelines (91).

- Level I evidence is based on randomized, controlled trials (or meta-analysis of such trials) of adequate size to ensure a low risk of incorporating false-positive or false-negative results.

- Level II evidence is based on randomized, controlled trials that are too small to provide level I evidence. These may show either positive trends that are not statistically significant or no trends, and are associated with a high risk of falsenegative results.

- Level III evidence is based on nonrandomized, controlled or cohort studies, case series, case-controlled studies or cross-sectional studies.

- Level IV evidence is based on the opinion of respected authorities or that of expert committees as indicated in published consensus conferences or guidelines.
- Level V evidence expresses the opinion of those individuals who have written and reviewed these guidelines, based on their experience, knowledge of the relevant literature and discussion with their peers.

Taking into account the differences in drug acquisition cost and spectrums of activity between erythromycin, clarithromycin and azithromycin, the following recommendations are made.

\section{First-line therapy}

- CAP in ambulatory patients (in combination with beta-lactam if modifying risk factors) (Level I)

- CAP in hospitalized patients in combination with beta-lactam (Level I)

- Single-dose treatment of uncomplicated nongonococcal urethritis or cervicitis (azithromycin $1 \mathrm{~g}$ orally) (Level I)

- For eradication of $H$ pylori infection in combination with a proton pump inhibitor and either amoxicillin or metronidazole (clarithromycin $500 \mathrm{mg}$ twice daily) (Level I)

- For treatment of infection with MAC (clarithromycin in combination with ethambutol with or without rifabutin) (Level I)

- For treatment of infection caused by susceptible nontuberculous mycobacteria (clarithromycin in combination with other agents) (Level IV)

- For prophylaxis of infection with MAC in HIV-infected patients azithromycin $1200 \mathrm{mg}$ once weekly (with or without rifabutin) (Level I)

\section{Alternate therapy}

- For treatment of infection with MAC (azithromycin in combination with ethambutol with or without rifabutin) (Level II)

- For prophylaxis of infection with MAC in HIV-infected patients (clarithromycin) (Level II)

- For patients experiencing or who have a history of severe gastrointestinal adverse effects while receiving any oral erythromycin formulation or conventional beta-lactam antibiotics (eg, penicillins and cephalosporins) for community-acquired respiratory tract infections (Level III)

- For patients who are likely to be nonadherent with standard erythromycin or conventional beta-lactam antibiotic regimen (Level IV)

- For prophylaxis of bacterial endocarditis for dental, oral, respiratory or gastrointestinal endoscopic procedures in patients with a history of beta-lactam hypersensitivity as recommended by the American Heart Association (92) (Level IV) 


\section{REFERENCES}

1. Bryskier A, Agouridas C, Chantot JF. Structure and activity. In: Neu H, Young LS, Zinner S, eds. The New Macrolides, Azalides, and Streptogramins. New York: Marcel Dekker Inc, 1993:3-8.

2. Peters DH, Clissold SP. Clarithromycin: A review of its antimicrobial activity, pharmacokinetic properties and therapeutic potential. Drugs 1992;44:117-64.

3. Wise R. The pharmacokinetics of azithromycin. Rev Contemp Pharmacother 1994;5:329-40.

4. Leclerq R, Courvalin P. Resistance to macrolides, azalides, and streptogramins. In: Neu H, Young LS, Zinner S, eds. The New Macrolides, Azalides, and Streptogramins. New York: Marcel Dekker Inc, 1993:33-40.

5. Widdowson CA, Klugman KP. Molecular mechanisms of resistance to commonly used non-betalactam drugs in Streptococcus pneumoniae. Semin Respir Infect 1999;1493:255-68.

6. Low DE. Trends and significance of antimicrobial resistance in respiratory pathogens. Curr Opin Infect Dis 2000;13:145-53.

7. Hoban DJ, Zhanel GG, Wierzbowski A, Karlowsky JA. Incidence of mefA and ermB among macrolide resistant Streptococcus pneumoniae (SPN) isolated in Canada during 1998 and 1999. 40th Interscience Conference on Antimicrobial Agents and Chemotherapy. Toronto, September 17 to 20, 2000. (Abst 2151)

8. Acar JF, Goldstein FW. In vitro activity against Gram-positive and Gram-negative bacteria. In: Neu H, Young LS, Zinner S, eds. The New Macrolides, Azalides, and Streptogramins. New York: Marcel Dekker Inc, 1993:13-24

9. Klemens SP, Cynamon MH. Activities of azithromycin and clarithromycin against nontuberculous mycobacteria in beige mice. Antimicrob Agents Chemother 1994;38:1455-9.

10. Rastogi N, Goh KS. Effect of pH on radiometric MICs of clarithromycin against 18 species of mycobacteria. Antimicrob Agents Chemother 1992;36:2841-2.

11. Cornish P. The new macrolides: azithromycin and clarithromycin. Can J Clin Pharmacol 1995;2:153-66.

12. Peters DH, Friedel HA, McTavish D. Azithromycin: A review of its antimicrobial activity, pharmacokinetic properties and clinical efficacy. Drugs 1992;44:750-99.

13. Nagata MJ, Tailor SA. Erythromycin-induced ototoxicity: A case report and review of the literature. Can J Hosp Pharm 2000;53:113-6.

14. Zithromax Product Monograph. Kirkland: Pfizer Canada Inc, August 6, 1999.

15. Amsden GW. Erythromycin, clarithromycin, and azithromycin: Are the differences real? Clin Ther 1996;18:56-72.

16. Foulds G, Chan KH, Johnson JT, Shephard RM, Johnson RB. Concentrations of azithromycin in human tonsillar tissue. Eur J Clin Microbiol Infect Dis 1991;10:853-6.

17. Piscitelli SC, Danziger LH, Rodvold KA. Clarithromycin and azithromycin: New macrolide antibiotics. Clin Pharm 1992;11:137-52.

18. Still JG, Hubbard WC, Poole JM, et al. Comparison of clarithromycin and penicillin VK suspensions in the treatment of children with streptococcal pharyngitis and review of currently available alternative antibiotic therapies. Pediatr Infect Dis J 1993;12(Suppl 3):S134-41.

19. Schaad UB, Heynen G. Evaluation of the efficacy, safety and toleration of azithromycin vs penicillin $\mathrm{V}$ in the treatment of acute streptococcal pharyngitis in children: results of a multicenter, open comparative study. The Swiss Tonsillopharyngitis Study Group. Pediatr Infect Dis J 1996;15:791-5.

20. McCarty J, Hedrick JA, Gooch WM. Clarithromycin suspension vs penicillin $\mathrm{V}$ suspension in children with streptococcal pharyngitis. Adv Ther 2000;17:14-26.

21. Bachand RT. A comparative study of clarithromycin and penicillin VK in the treatment of outpatients with streptococcal pharyngitis. J Antimicrob Chemother 1991;27:75S-82S.

22. Pacifico L, Scopetti F, Ranucci A, Pataracchia M, Savignoni F, Chiesa C. Comparative efficacy and safety of 3-day azithromycin and 10-day penicillin $\mathrm{V}$ treatment of group A beta-hemolytic streptococcal pharyngitis in children. Antimicrob Agents Chemother 1996;40:1005-8.

23. Still JG. Management of pediatric patients with group A beta-hemolytic Streptococcus pharyngitis: treatment options. Pediatr Infect Dis J 1995;14:S57-61.

24. Still JG. Treatment of streptococcal pharyngitis in children with five days of azithromycin suspension. 34th Interscience Conference on Antimicrobial Agents and Chemotherapy. Orlando, October 4 to 7 , 1994. (Abst)

25. Calhoun $\mathrm{KH}$, Hokanson JA. Multicenter comparison of clarithromycin and amoxicillin in the treatment of acute maxillary sinusitis. Arch Fam Med 1993;2:837-40.

26. Dubois J, Saint-Pierre C, Tremblay C. Efficacy of clarithromycin vs amoxicillin/clavulanate in the treatment of acute maxillary sinusitis. Ear Nose Throat J 1993;72:804-10.

27. Amin NM, Breadon G. An open-label, non-comparative study to evaluate the efficacy, safety, and tolerability of azithromycin in the treatment of patients with acute sinusitis. Clin Ther 1995;17:701-7.

28. Clement PA, de Gandt JB. A comparison of the efficacy, tolerability and safety of azithromycin and co-amoxiclav in the treatment of sinusitis in adults. J Int Med Res 1998;26:66-75.

29. Principi N. Multicentre comparative study of the efficacy and safety of azithromycin compared with amoxicillin/clavulanic acid in the treatment of paediatric patients with otitis media. Eur J Clin Microbiol Infect Dis 1995;14:669-76.

30. Dagan R, Johnson CE, McLinn S, et al. Bacteriologic and clinical efficacy of amoxicillin/clavulanate vs azithromycin in acute otitis media. Pediatr Infect Dis J 2000;19:95-104.

31. Dagan R, Leibovitz E, Fliss DM, et al. Bacteriologic efficacies of oral azithromycin and oral cefaclor in treatment of acute otitis media in infants and young children. Antimicrob Agents Chemother 2000;44:43-50.

32. Pukander JS, Jero JP, Kaprio EA, Sorri MJ. Clarithromycin vs amoxicillin suspensions in the treatment of pediatric patients with acute otitis media. Pediatr Infect Dis J 1993;12(Suppl 3):S118-21.

33. Coles SJ, Addlestone MB, Kamdar MK, Macklin JL. A comparative study of clarithromycin and amoxycillin suspensions in the treatment of pediatric patients with acute otitis media. Infection 1993;21:272-8.

34. Aspin MM, Hoberman A, McCarty J, et al. Comparative study of the safety and efficacy of clarithromycin and amoxicillin-clavulanate in the treatment of acute otitis media in children. J Pediatr 1994;125:136-41.

35. McCarty JM, Phillips A, Wilsanen R. Comparative safety and efficacy of clarithromycin and amoxicillin/clavulanate in the treatment of acute otitis media in children. Pediatr Infect Dis J 1993:12(Suppl 3):S122-7.

36. Gooch WM, Gan VN, Coirder WT, Khurana CM, Andrews WP. Clarithromycin and cefaclor suspensions in the treatment of acute otitis media in children. Pediatr Infect Dis J 1993;12(Suppl 3):S128-33.

37. Wettengel R, Vetter N, Waardenburg FA. Clarithromycin versus cefaclor for the treatment of mild-to-moderate acute bacterial bronchitis. J Antimicrob Chemother 1993;31:963-72.

38. Adam D. Clarithromycin $250 \mathrm{mg}$ bid for 5 or 10 days in the treatment of adult patients with purulent bronchitis. Infection 1993:21:265-71.

39. Chodosh S, Schreurs A, Siami G, et al. Efficacy of oral ciprofloxacin vs clarithromycin for treatment of acute bacterial exacerbations of chronic bronchitis. The Bronchitis Study Group. Clin Infect Dis 1998;27:730-8.

40. Anzueto A, Niederman MS, Tillotson GS. Etiology, susceptibility, and treatment of acute bacterial exacerbations of complicated chronic bronchitis in the primary care setting: ciprofloxacin $750 \mathrm{mg}$ bid versus clarithromycin $500 \mathrm{mg}$ bid. Bronchitis Study Group. Clin Ther 1998;20:885-900.

41. Neu HC, Chick TW. Efficacy and safety of clarithromycin compared to cefixime as outpatient treatment of lower respiratory tract infections. Chest 1993;104:1393-9.

42. Fong IW, Laforge J, Dubois J, Small D, Grossman R, Zakhari R. Clarithromycin versus cefaclor in lower respiratory tract infections. The Canadian Bronchitis Study Group. Clin Invest Med 1995;18:131-8.

43. Bradbury F. Comparison of azithromycin versus clarithromycin in the treatment of patients with lower respiratory tract infections. J Antimicrob Chemother 1993;31(Suppl E):153-62.

44. Biebuyck XA. Comparison of azithromycin and co-amoxiclav in the treatment of acute tracheobronchitis and acute infectious exacerbations of chronic bronchitis in adults. Azithromycin Study Group. J Int Med Res 1996;24:407-18.

45. Aoyama T, Sunadawa K, Iwata S, Takeuchi Y, Fujii R. Efficacy of shortterm treatment of pertussis with clarithromycin and azithromycin. J Pediatr 1996;129:761-4.

46. Bace A, Zrnic T, Begovac J, Kuzmanovic N, Culig J. Short-term treatment of pertussis with azithromycin in infants and young children. Eur J Clin Microbiol Infect Dis 1999;18:296-8.

47. Jang TN, Liu CY, Wang FD, Yang SP, Fung CP. A randomized comparative study on the safety and efficacy of clarithromycin and erythromycin in treating community-acquired pneumonia. Chin Med J (Engl) 1995;55:302-6

48. Chien SM, Pichotta P, Siepman N, Chan CK. Treatment of community-acquired pneumonia. A multicenter, double-blind, randomized study comparing clarithromycin with erythromycin. Canada-Sweden Clarithromycin-Pneumonia Study Group. Chest 1993;103:697-701.

49. Rizzato G, Montemurro L, Fraioli P, et al. Efficacy of a three day course of azithromycin in moderately severe community-acquired pneumonia. Eur Respir J 1995;8:398-402.

50. Bohte R, van't Wout JW, Lobatto S, et al. Efficacy and safety of 
azithromycin versus benzylpenicillin or erythromycin in communityacquired pneumonia. Eur J Clin Microbiol Infect Dis 1995;14:182-7.

51. Vergis EN, Indorf A, File T, et al. Azithromycin vs cefuroxime plus erythromycin for empirical treatment of community-acquired pneumonia in hospitalized patients. Arch Intern Med 2000;160:1294-300.

52. Plouffe J, Schwartz DB, Kolokathis A, et al. Clinical efficacy of intravenous followed by oral azithromycin monotherapy in hospitalized patients with community-acquired pneumonia. The Azithromycin Intravenous Clinical Trials Group. Antimicrob Agents Chemother 2000;44:1796-802.

53. Uzun O, Hayran M, Akova M, Gur D, Akalin HE. Efficacy of a three-day course of azithromycin in the treatment of community-acquired pneumococcal pneumonia. J Chemother 1994;6:53-7.

54. Harris JA, Kolokathis A, Campbell M, Cassell GH, Hammerschlag MR. Safety and efficacy of azithromycin in the treatment of communityacquired pneumonia in children. Pediatr Infect Dis J 1998;17:865-71.

55. Parish LC. Clarithromycin in the treatment of skin and skin structure infections: two multicenter clinical studies. Clarithromycin Study Group. Int J Dermatol 1993;32:528-32.

56. Herbert AA, Still JG, Reuman PD. Comparative safety and efficacy of clarithromycin and cefadroxil suspensions in the treatment of mild to moderate skin and skin structure infections in children. Pediatr Infect Dis J 1993;12(Suppl 3):S112-7.

57. Lassus A. Comparative studies of azithromycin in skin and soft-tissue infections and sexually transmitted infections by Neisseria and Chlamydia species. J Antimicrob Chemother 1990;25(Suppl A):115-21.

58. Daniel R. Azithromycin, erythromycin and cloxacillin in the treatment of infections of skin and associated soft tissues. European Azithromycin Study Group. J Int Med Res 1991;19:433-45.

59. Kiani R. Double-blind, double-dummy comparison of azithromycin and cephalexin in the treatment of skin and skin structure infections. Eur J Clin Microbiol Infect Dis 1991;10:880-4

60. Mallory SB. Azithromycin compared with cephalexin in the treatment of skin and skin structure infections. Am J Med 1991;91:36S-9S.

61. Montero L. A comparative study of the efficacy, safety and tolerability of azithromycin and cefaclor in the treatment of children with acute skin and/or soft tissue infections. J Antimicrob Chemother 1996;37(Suppl C):125-31.

62. Steingrimsson O, Olafsson JH, Thorarinsson H, Ryan RW, Johnson RB, Tilton RC. Single dose azithromycin treatment of gonorrhea and infections caused by $C$ trachomatis and $U$ urealyticum in men. Sex Transm Dis 1994;21:43-6.

63. Thorpe EM, Stamm WE, Hook EW, et al. Chlamydial cervicitis and urethritis: single dose treatment compared with doxycycline for seven days in community based practices. Genitourin Med 1996;72:93-7.

64. Stamm WE, Hicks CB, Martin DH, et al. Azithromycin for empirical treatment of the nongonococcal urethritis syndrome in men. A randomized double-blind study. JAMA 1995;274:545-9.

65. Stein GE, Mummaw NL, Havlichek DH. A preliminary study of clarithromycin versus doxycycline in the treatment of nongonococcal urethritis and mucopurulent cervicitis. Pharmacotherapy 1995; 15:727-31.

66. Wehbeh HA, Ruggeirio, Shahem S, Lopez G, Ali Y. Single-dose azithromycin for chlamydia in pregnant women. J Reprod Med 1998;43:509-14.

67. Pierce M, Crampton S, Henry D, et al. A randomized trial of clarithromycin as prophylaxis against disseminated Mycobacterium avium complex infection in patients with advanced acquired immunodeficiency syndrome. N Engl J Med 1996;335:384-91.

68. Benson CA, Williams PL, Cohn DL, et al. Clarithromycin or rifabutin alone or in combination for primary prophylaxis of Mycobacterium avium complex disease in patients with AIDS: A randomized, double-blind, placebo-controlled trial. The AIDS Clinical Trials Group. J Infect Dis 2000;181:1289-97.

69. Havlir DV, Dube MP, Sattler FR, et al. Prophylaxis against disseminated Mycobacterium avium complex with weekly azithromycin, daily rifabutin, or both. California Collaborative Treatment Group. N Engl J Med 1996;335:392-8.

70. Chaisson RE, Benson CA, Dube MP, et al. Clarithromycin therapy for bacteremic Mycobacterium avium complex disease. A randomized, double-blind, dose-ranging study in patients with AIDS. AIDS Clinical Trials Group Protocol 157 Study Team. Ann Intern Med 1994;121:905-11.

71. Dautzenberg B, Saint Marc T, Meyohas MC, et al. Clarithromycin and other antimicrobial agents in the treatment of disseminated Mycobacterium avium infections in patients with acquired immunodeficiency syndrome. Arch Intern Med 1993;153:368-72.

72. Wallace RJ, Brown BA, Griffith DE, et al. Initial clarithromycin monotherapy for Mycobacterium avium complex lung disease. Am J Respir Crit Care Med 1994;149:1335-41.
73. Heifets LB. Clarithromycin against Mycobacterium avium complex infections. Tuberc Lung Dis 1996;77:19-26.

74. Dube MP, Sattler FR, Torriani FJ, et al. California Collaborative Treatment Group. A randomized evaluation of ethambutol for prevention of relapse and drug resistance during treatment of Mycobacterium avium complex bacteremia with clarithromycin-based combination therapy. J Infect Dis 1997;176:1225-32.

75. Shafran SD, Singer J, Zarowny DP, et al. A comparison of two regimens for the treatment of Mycobacterium avium complex bacteremia in AIDS: rifabutin, ethambutol, and clarithromycin versus rifampin, ethambutol, clofazimine, and ciprofloxacin. Canadian HIV Trials Network Protocol 010 Study Group. N Engl J Med 1996;335:377-83.

76. Ward TT, Rimland D, Kauffman C, Huycke M, Evans TG, Heifets L. Randomized, open-label trial of azithromycin plus ethambutol vs clarithromycin plus ethambutol as therapy for Mycobacterium avium complex bacteremia in patients with human immunodeficiency virus infection. Veterans Affairs HIV Research Consortium. Clin Infect Dis 1998;27:1278-85.

77. Diagnosis and treatment of disease caused by nontuberculous mycobacteria. The American Thoracic Society. Am J Respir Crit Care Med 1997;156:S1-25.

78. Wallace RJ, Tanner D, Brennan PJ, Brown BA. Clinical trial of clarithromycin for cutaneous (disseminated) infection due to Mycobacterium chelonae. Ann Intern Med 1993;119:482-6.

79. Chan GP, Garcia-Ignacio BY, Chavez VE, et al. Clinical trial of clarithromycin for lepromatous leprosy. Antimicrob Agents Chemother 1994;38:515-7.

80. Schmid CH, Whiting G, Cory D, Ross SD, Chalmers TC. Omeprazole plus antibiotics in the eradication of Helicobacter pylori infection: a meta-regression analysis of randomized, controlled trials. Am J Ther 1999;6:25-36.

81. Laheij RJ, Rossum LG, Jansen JB, Straatman H, Verbeek AL. Evaluation of treatment regimens to cure Helicobacter pylori infection a meta-analysis. Aliment Pharmacol Ther 1999;13:857-64.

82. Huang J, Hunt RH. The importance of clarithromycin dose in the management of Helicobacter pylori infection: a meta-analysis of triple therapies with a proton pump inhibitor, clarithromycin and amoxycillin or metronidazole. Aliment Pharmacol Ther 1999;13:719-29.

83. Unge P. Antimicrobial treatment of $H$ pylori infection - a pooled efficacy analysis of eradication therapies. Eur J Surg Suppl 1998;582:16-26

84. Taylor JL, Zagari M, Murphy K, Freston JW. Pharmacoeconomic comparison of treatments for the eradication of Helicobacter pylori. Arch Intern Med 1997;157:87-97.

85. Unge P. Review of Helicobacter pylori eradication regimens. Scand J Gastroenterol Suppl 1996;215:74-81.

86. Reed MD, Blumer JL. Azithromycin: a critical review of the first azalide antibiotic and its role in pediatric practice. Pediatr Infect Dis ] 1997;16:1069-83.

87. Newer macrolides offer some advantages over erythromycin. Drug Ther Perspect 1995;5:5-9.

88. Luke DR, Foulds G. Toleration of intravenous azithromycin. Ann Pharmacother 1997; 31:965-9.

89. Bizjak ED, Haug MT, Schilz RJ, Sarodia BD, Dresing JM. Intravenous azithromycin-induced ototoxicity. Pharmacotherapy 1999;19:245-8.

90. Rapp RP. Pharmacokinetics and pharmacodynamics of intravenous and oral azithromycin: Enhanced tissue activity and minimal drug interactions. Ann Pharmacother 1998;32:785-93.

91. The Steering Committee on Clinical Practice Guidelines for the Care and Treatment of Breast Cancer. Clinical practice guidelines for the care and treatment of breast cancer: A Canadian consensus document. CMAJ 1998;158(3 Suppl):52.

92. Dajani AS, Taubert KA, Wilson W, et al. Prevention of bacterial endocarditis. Recommendations by the American Heart Association. JAMA 1997;277:1794-801.

93. Ridgway GL. In vitro activity against Mycoplasma spp and intracellular organisms. In: Neu H, Young LS, Zinner S, eds. The New Macrolides, Azalides, and Streptogramins. New York: Marcel Dekker Inc, 1993:25-30.

94. Yew WW, Piddock LJV, Li MSK, Lyon D, Chan CY, Cheng AFB. In-vitro activity of quinolones and macrolides against mycobacteria. J Antimicrob Chemother 1994;34:343-51.

95. Biaxin Product Monograph. Saint-Laurent: Abbott Laboratories Limited, September 24, 1998.

96. Tatro DS, ed. Drug Interactions Facts. St Louis: Facts and Comparisons, 1999.

97. Ontario Drug Benefit Formulary, No 37, March 2001. Toronto: The Drug Quality and Therapeutics Committee, Ministry of Health and Long Term Care, Publications Toronto, 2001. 


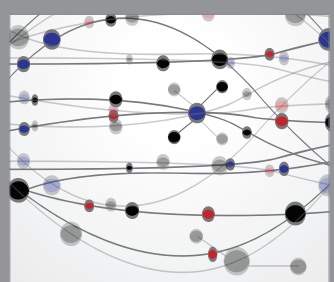

The Scientific World Journal
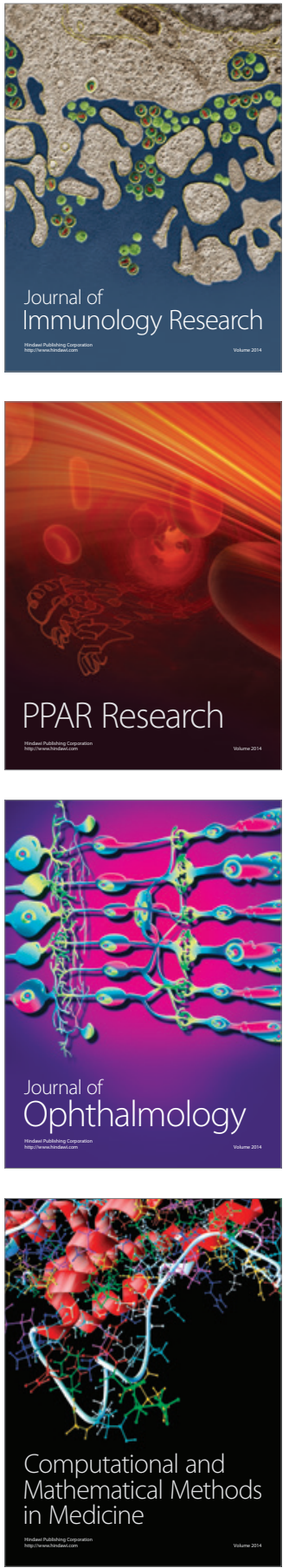

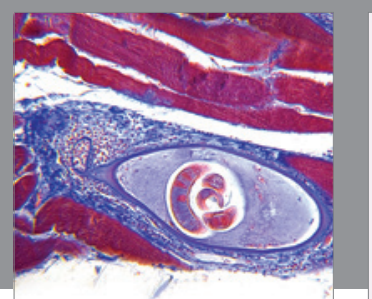

Gastroenterology Research and Practice



\section{Hindawi}

Submit your manuscripts at

http://www.hindawi.com

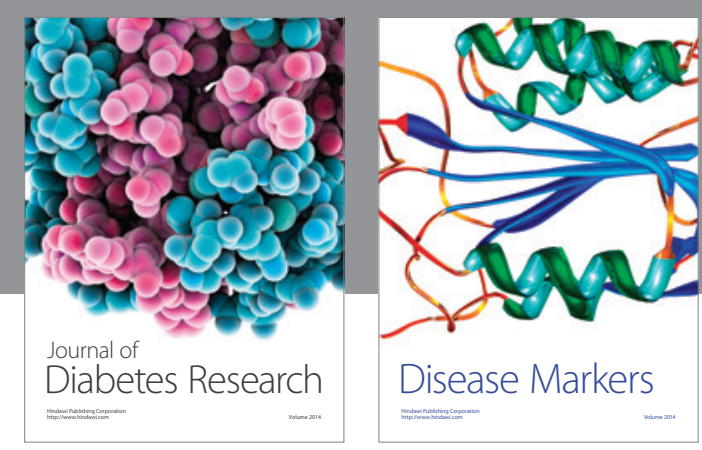

Disease Markers
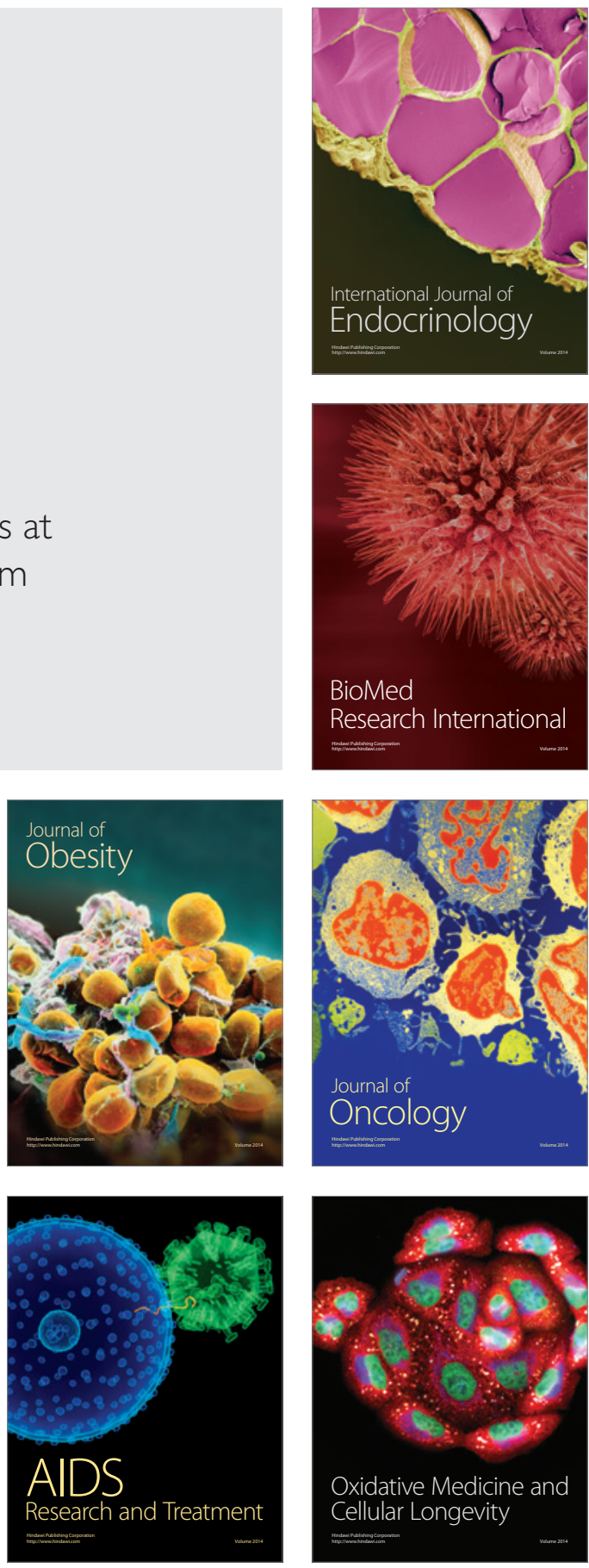